\title{
Avaliação de sintomas na distrofia muscular de Duchenne: uma estratégia de cuidado paliativo
}

\section{Evaluation of symptoms in Duchenne muscular dystrophy: a palliative care strategy}

(D) Josiane Rosires Pavão ${ }^{1}$ (DMariana Angélica de Souza ${ }^{1}$, (DElisângela Aparecida da Silva Lizzi², (DCláudia Ferreira da Rosa Sobreira ${ }^{3}$, (D)Thais Cristina Chaves ${ }^{1}$, (D)Ana Claudia Mattiello-Sverzut ${ }^{1}$

\section{RESUMO}

A avaliação de sintomas na distrofia muscular de Duchenne (DMD) permite o adequado manejo terapêutico e o Edmonton Symptom Assessment System (ESAS) possui esta função: avaliar simultaneamente múltiplos sintomas de pacientes em cuidados paliativos (dor, cansaço, sonolência, náusea, apetite, falta de ar, depressão, ansiedade e bem-estar). Objetivo: Verificar se pacientes com DMD entendem os termos do ESAS e são capazes de graduar seus sintomas por este instrumento. Métodos: 10 pacientes com DMD foram avaliados transversalmente em relação à: compreensão dos itens do ESAS, caracterização dos sintomas (pelo ESAS e Escala de Faces) e medida da função motora. A graduação de sintomas do paciente, pelo ESAS, foi realizada também pelo avaliador. Os dados foram analisados descritivamente e por meio do coeficiente de correlação de Spearman. Resultados: Todos os pacientes compreenderam os sintomas dor, cansaço, sonolência, depressão (tristeza) e bem-estar, já os sintomas náusea, apetite, falta de ar e ansiedade não foram compreendidos por todos. A média geral de todos os sintomas avaliados pela escala ESAS foi abaixo de 5 pontos. Entre os resultados da escala ESAS e Escala de faces, houve correlação forte para os sintomas "depressão" $(r=0,64)$ e "ansiedade" $(r=0,65)$. Houve correlação perfeita $(r=1,0)$ entre ESAS preenchida pelo paciente e pelo avaliador para os itens "depressão" e "ansiedade" e correlação forte $(r=0,82)$ para "sonolência". Conclusão: Pacientes com DMD entenderam os termos do ESAS e graduaram seus sintomas por este instrumento, portanto, não há necessidade de alteração dos termos do ESAS para avaliação de pacientes com DMD.

Palavras-chave: Distrofia Muscular de Duchenne, Cuidados Paliativos, Avaliação de Sintomas

${ }^{1}$ Departamento de Ciências da Saúde, Faculdade de Medicina de Ribeirão Preto da Universidade de São Paulo - FMRP-USP

2 Departamento de Matemática, Universidade Tecnológica Federal do Paraná - UTFPR

${ }^{3}$ Departamento de Neurociências e Ciências do Comportamento, Faculdade de Medicina de Ribeirão Preto da Universidade de São Paulo FMRP-USP

Correspondência

Ana Claudia Mattiello-Sverzut

E-mail: acms@fmrp.usp.br

Submetido: 30 Junho 2020

Aceito: 17 Agosto 2020

\section{Como citar}

Pavão JR, Souza MA, Lizzi EAS, Sobreira CFR, Chaves TC, Mattiello-Sverzut AC. Avaliação de sintomas na distrofia muscular de Duchenne: uma estratégia de cuidado paliativo. Acta Fisiatr. 2020;27(2):89-94.

DOI: 10.11606/issn.2317-0190.v27i2a171476

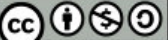

BY NC SA

Este trabalho está licenciado com uma licença

Creative Commons - Atribuição 4.0 Internacional

\section{ABSTRACT}

Symptom assessment, in Duchenne Muscular Dystrophy (DMD), allows an adequate treatment, and the Edmonton Symptom Assessment System (ESAS) assess it: evaluating clinical problems of patients in palliative care (pain, tiredness, drowsiness, nausea, appetite, shortness of breath, depression, anxiety, and well-being). Objective: To verify if patients with DMD understand the terms of the ESAS and if their symptoms could be assessed using this instrument. Methods: Ten patients with DMD were cross-sectional evaluated in relation to the understanding of the ESAS items, capacity to describe symptom (using the ESAS and the Faces Scale) and the Motor Function Measure. The patient's symptom by ESAS was also classified by evaluator. A descriptive and correlation (Spearman's correlation coefficient) analysis of data was performed. Results: All patients understood the symptoms of pain, tiredness, drowsiness, depression (sadness), and wellbeing. However, some patients did not understand the symptoms of nausea, appetite, shortness of breath. The general mean of all symptoms assessed by the ESAS was below 5 points. For the symptom 'depression' and 'anxiety', a strong correlation was found between the results of the ESAS and the Face scale ( $r=0.64$, and $r=0.65$, respectively). Additionally, a perfect and strong correlation, respectively, was found between the ESAS completed by the patient and the evaluator for the items "depression'," and 'anxiety' ( $r=$ 1.0)" and a 'drowsiness' ( $r=0.82)$. Conclusion: DMD patients understood the ESAS terms and graded their symptoms using this instrument. Therefore, it is not necessary to change the ESAS terms to assess patients with DMD.

Keywords: Muscular Dystrophy, Duchenne, Palliative Care, Symptom Assessment 


\section{INTRODUÇÃO}

A Distrofia Muscular de Duchenne (DMD) é caracterizada clinicamente por fraqueza muscular progressiva, levando a deficiências físicas e incapacitantes que levam a morte na segunda ou terceira década de vida. ${ }^{1}$ Além dessas anormalidades musculoesqueléticas, esses pacientes também podem apresentar alta carga sintomática como dor crônica, ${ }^{2}$ fadiga, ansiedade, depressão, ${ }^{3}$ cãibras musculares, dispneia, constipação, entre outros. ${ }^{4}$ É importante destacar que a avaliação inadequada de sintomas pode acarretar em aumento do sofrimento tanto do paciente quanto da família e implicar em prejuízos na qualidade de vida. ${ }^{4,5}$

O alívio de sintomas desagradáveis constitui o primeiro princípio do Cuidado Paliativo, cuja definição é "uma abordagem que melhora a qualidade de vida de pacientes (adultos e crianças) e famílias que enfrentam problemas associados a doenças que ameaçam a vida. Previne e alivia o sofrimento por meio da identificação precoce, avaliação correta e tratamento da dor e outros problemas físicos, psicossociais ou espirituais". ${ }^{6}$ Por ser uma doença crônica e progressiva, sem a possibilidade de tratamento curativo, a DMD se qualifica para receber esses cuidados desde o momento do diagnóstico. ${ }^{7}$

Nesse contexto, conhecer o perfil sintomático dos pacientes com DMD pode favorecer a proposição de planos terapêuticos mais completos e resolutivos.

Existem vários instrumentos que se propõem a avaliar os sintomas de pacientes com indicação de cuidados paliativos, sendo um deles o Edmonton Symptom Assessment System (ESAS). ${ }^{8,9}$ Seu uso é comum em pacientes adultos oncológicos, com doenças renais, ${ }^{10}$ insuficiência cardíaca, ${ }^{11}$ distúrbios pulmonares, ${ }^{12}$ doenças hepáticas ${ }^{13}$ e anemia falciforme. ${ }^{14}$ Essa ferramenta, considerada de fácil aplicação, avalia simultaneamente nove sintomas físicos e psicológicos (dor, cansaço, náusea, depressão, ansiedade, sonolência, apetite, falta de ar e bem-estar), graduando-os de zero a dez. ${ }^{8,9}$

Pode ser preenchida pelo paciente ou até mesmo pela percepção do cuidador ou do profissional da saúde sobre o paciente. ${ }^{8} \mathrm{E}$, outra vantagem é que já existe sua versão brasileira traduzida e validada. ${ }^{9}$

De modo geral, os sintomas na DMD são avaliados de forma isolada por diferentes instrumentos, como por exemplo: Escala Faces Revisada para avaliar dor, ${ }^{15}$ Escala Multidimensional de Fadiga para fadiga, ${ }^{16}$ Escala de Borg Modificada para dispneia, ${ }^{17}$ Escala de Distúrbios do Sono em Crianças para sonolência, ${ }^{18}$ Escala Hospitalar de Ansiedade e Depressão para avaliar ansiedade e depressão ${ }^{3}$ e Escala Baxter Retching Faces para náusea. ${ }^{19}$

Importante destacar que não existem relatos na literatura de instrumentos que avaliem alterações de apetite em crianças. Por permitir o reconhecimento de um conjunto de sintomas que podem estar presentes na vida desses pacientes, - ESAS pode fornecer subsídios para tomada de decisão e auxílio na elaboração do plano terapêutico integral. ${ }^{5}$

Como esse instrumento nunca foi utilizado em pacientes com DMD e foi utilizado apenas uma vez na população pediátrica com diagnóstico oncológico, ${ }^{20}$ faz-se necessário inicialmente investigar se os pacientes (crianças e adolescentes) compreendem os termos utilizados no instrumento.
Questiona-se: um paciente de 8 anos, com DMD, sabe relatar se está com falta de ar ou náusea, por exemplo? E qual seria a intensidade desse desconforto? Se a equipe de saúde não tem respostas assertivas para essas questões na DMD, não há como propor uma conduta adequada. Assim, destaca-se que uma avaliação inadequada pode ser um entrave na abordagem integral e resolutiva.

\section{OBJETIVO}

\section{Geral}

Verificar se pacientes (crianças e adolescentes) com DMD entendem os termos do ESAS e são capazes de graduar seus sintomas por este instrumento.

\section{Específicos}

Descrever e correlacionar a pontuação relatada pelo paciente no ESAS com a pontuação na Escala de Faces para cada sintoma avaliado. Descrever e correlacionar a pontuação relatada pelo paciente e pelo avaliador para cada sintoma avaliado pelo ESAS.

\section{MÉTODOS}

Este estudo consistiu de uma amostra de conveniência de 10 pacientes do Ambulatório de Miopatias Infantis (FIC-138) do Centro de Reabilitação do Hospital das Clínicas da Faculdade de Medicina de Ribeirão Preto da Universidade de São Paulo (HCFMRP-USP). Para participar do estudo os pacientes precisavam: a) ter diagnóstico confirmado de $\mathrm{DMD}, \mathrm{b}$ ) ter idade entre 8 e 13 anos, c) consentir à pesquisa mediante assinatura do termo de consentimento livre e esclarecido e termo de assentimento.

Foram excluídos os pacientes que demonstraram incapacidade em compreender os comandos dados pelo avaliador. Este estudo foi aprovado pelo Comitê de Ética do HCFMRP (CAEE no: 16348819.3.0000.5440).

Foi realizada uma entrevista individual com cada paciente para avaliar o entendimento dos termos da escala (dor, cansaço, náusea, tristeza, ansiedade, sonolência, apetite, falta de ar e bem-estar). Inicialmente, um avaliador previamente treinado em relação ao uso do instrumento questionou se o paciente conhecia os termos e para isso foi feita a seguinte pergunta "você já ouviu a palavra dor?" assim foi feito com todos os sintomas. Caso o paciente respondesse que não conhecia a palavra, era feita uma rápida explicação afim de verificar se o mesmo utilizava algum outro termo para o referido sintoma, além disso, os pais ou responsáveis também eram questionados se havia algum outro termo que era utilizado e se o paciente já havia apresentado alguma queixa em relação ao sintoma.

Após a verificação inicial da compreensão dos termos, o paciente foi orientado a escolher um número entre 0 (zero) e 10 (dez) para cada um dos noves sintomas avaliados pelo ESAS, sendo 0 (zero) ausência completa e 10 (dez) em sua máxima intensidade. $O$ paciente recebeu o seguinte comando verbal: "zero é nada, você está sem dor e dez é a pior dor que você sentiu na sua vida. Que nota você dá para a sua dor agora?"

Esse comando foi realizado para todos os demais sintomas avaliados pela escala. 
Simultaneamente, o avaliador também preencheu o ESAS em relação à sua percepção sobre os sintomas do paciente. Não há descrição na literatura em quais parâmetros ou sinais clínicos os profissionais devem se basear para realizar a referida pontuação. Neste estudo, o avaliador, previamente treinado na anamnese e semiologia do paciente com DMD, pontuou o ESAS baseado na observação do comportamento do paciente levando em consideração as expressões faciais e corporais do mesmo.

Posteriormente, para avaliar se o paciente conseguia quantificar de 0 a 10 os sintomas avaliados pela escala, foi utilizada uma Escala de Faces. ${ }^{3}$ Para isso o paciente graduou novamente cada sintoma utilizando esta escala mediante o seguinte comando verbal: "qual dessas imagens representa a dor que você está sentindo agora?" e assim foi feito para todos os sintomas. Sendo que o paciente deveria apontar para aquela que melhor representasse o seu sintoma no momento.

Para a avaliação de sintomas foram utilizados o ESAS-r, seguindo as recomendações dos autores que realizaram a validação e adaptação para o português, ${ }^{21}$ e a uma Escala de Faces traduzida e adaptada para população brasileira. ${ }^{22}$

Já os dados da medida da função motora (MFM) foram adquiridos no banco de dados do FIC138, sendo que o intervalo de avaliação entre MFM e ESAS foi de, no máximo, 6 meses (dois pacientes, P6 e P10, não possuem dados de MFM que contemple esse período). Na rotina do ambulatório, a MFM é aplicada seguindo as recomendações do manual.

A mesma é composta por 32 itens agrupados em três dimensões: Dimensão 1 (avalia a posição em pé e transferências), Dimensão 2 (avalia a função motora axial e proximal) e da Dimensão 3 (avalia a função motora distal), e cada item é graduado em escores de 0 a 3 de acordo com as habilidades do paciente. ${ }^{23}$

Inicialmente, foi realizada uma análise descritiva dos dados, utilizando as médias e desvios-padrão. Foi utilizado o coeficiente de correlação de Spearman para mensurar a correlação entre: (1) as pontuações obtidas na escala ESAS-r com as pontuações obtidas pela Escala de Faces e (2) as pontuações obtidas na escala ESAS-r pelo paciente e as pontuações obtidas na escala ESAS-r pelo avaliador, pois como se trata de escores quantitativos e discretizados, optou-se por um método não - paramétrico $^{24}$ para viabilizar essa mensuração. Nestas análises considerou-se um nível de significância de $5 \%$ e os ajustes foram obtidos no software SAS (versão 9.2).

Todas as análises foram realizadas considerando a amostra total de 10 pacientes, com exceção da análise descritiva dos dados da MFM que foi referente à análise de dados de 8 pacientes.

\section{RESULTADOS}

Como mencionado, esse estudo foi composto por uma amostra de conveniência, cujo fluxograma de recrutamento está disponível na Figura 1 e a caracterização na Tabela 1.

Considerando toda a amostra, a média de idade foi 10,5 $(\mathrm{DP}=2,40)$ anos, a média da idade de diagnóstico foi 7,50 (DP= 1,17) anos, todos faziam uso de corticoides e três eram cadeirantes (Tabela 1).

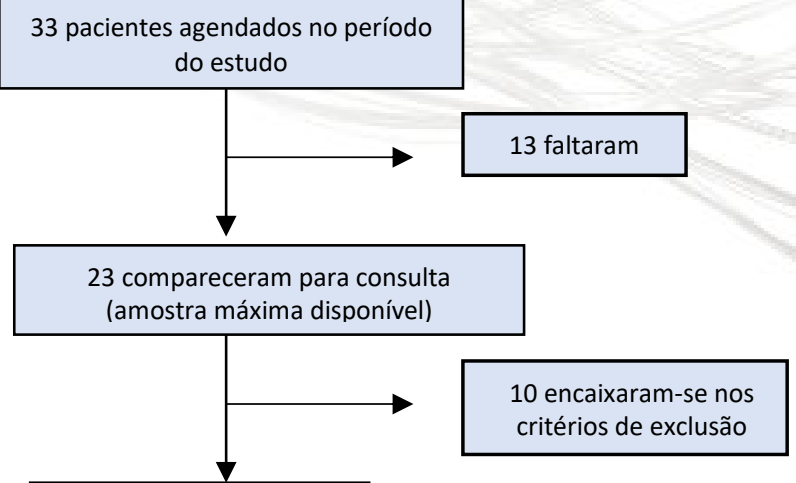

10 pacientes avaliados

Figura 1. Recrutamento da amostra

Em relação à pontuação da MFM, o escore médio total dos 8 pacientes que possuíam essa avaliação foi de 73,95\% (DP= 19,82). Os escores médios da Dimensão 1, Dimensão 2 e da Dimensão 3 foram respectivamente: 48,71\% ( $D P=33,00)$; $92,35 \% \quad(D P=14,14)$ e $89,28 \% \quad(D P=11,59) \quad($ Tabela 1). Compreensão dos itens do ESAS.

\section{Compreensão dos itens do ESAS}

Os 10 pacientes completaram o ESAS e os sintomas dor, cansaço, sonolência, depressão (tristeza) e bem-estar foram compreendidos por todos. Já os sintomas náusea, apetite, falta de ar e ansiedade não foram compreendidos por todos os pacientes (Tabela 2). Todos os sintomas foram completamente compreendidos, sem necessidade de explicação adicional por quatro pacientes (P1, P6, P8 e P10).

\section{Pontuações dos sintomas nas escalas ESAS e Escala de Faces}

A Tabela 3 mostra a pontuação de cada sintoma dos 10 pacientes em ambos os instrumentos utilizados e a correlação entre os resultados desses instrumentos, para cada sintoma avaliado.

A análise da pontuação dos sintomas nas escalas ESAS e Escala de Faces mostrou que a média geral de todos os sintomas avaliados pela escala ESAS foi baixa, ou seja, a média das pontuações dos pacientes foi abaixo de 5 para todos os sintomas, caracterizando os sintomas, quando presentes, em intensidade leve. A correlação entre os resultados da escala ESAS preenchida pelo paciente e Escala de Faces mostrou que os itens "sonolência" e "apetite" apresentaram correlação moderada, $r=0,50$ e $r=0,57$, respectivamente, enquanto os itens "depressão" e "ansiedade" apresentaram correlação forte, $r=0,64$ e $r=0,65$ respectivamente. Os itens "dor", "náusea" e "bem-estar" não foram possíveis correlacionar devido à ausência de variabilidade nas respostas.

Simultaneamente à obtenção dos sintomas por meio da resposta dos pacientes, o avaliador do estudo pontuou cada sintoma de acordo com sua percepção do paciente. A correlação entre os resultados da escala ESAS preenchida pelo paciente e pelo avaliador mostrou que os itens "depressão" e "ansiedade" apresentaram correlação perfeita, $r=1,00$ e $r=$ 1,00 respectivamente. Para o item "sonolência" apresentou correlação forte $(r=0,82)$. Os demais itens não foram possíveis correlacionar devido à ausência de variabilidade nas respostas. 
Tabela 1. Caracterização da amostra

\begin{tabular}{|c|c|c|c|c|c|c|c|c|c|}
\hline \multirow[b]{2}{*}{ Paciente } & \multirow[b]{2}{*}{ Idade (anos) } & \multirow{2}{*}{$\begin{array}{c}\text { Idade de } \\
\text { diagnóstico da } \\
\text { doença (anos) }\end{array}$} & \multirow{2}{*}{$\begin{array}{c}\text { Uso de corticoide } \\
\text { / órtese? }\end{array}$} & \multirow[b]{2}{*}{ Fisioterapia? } & \multirow[b]{2}{*}{ Mobilidade } & \multicolumn{4}{|c|}{ MFM } \\
\hline & & & & & & $\begin{array}{l}\text { D1 } \\
(\%)\end{array}$ & $\begin{array}{l}\text { D2 } \\
(\%)\end{array}$ & $\begin{array}{l}\text { D3 } \\
(\%)\end{array}$ & $\begin{array}{c}\text { Escore total } \\
(\%)\end{array}$ \\
\hline 1 & 8 & 6 & S/S; D & Sim & Deambulador & 79,48 & 100 & 100 & 91,66 \\
\hline 2 & 8 & 6 & S / S; No & Sim & Deambulador & 46,15 & 94,44 & 80,95 & 71,87 \\
\hline 3 & 8 & 6 & S / S; D e No & Sim & Deambulador & 23,07 & 91,66 & 85,71 & 62,5 \\
\hline 4 & 8 & 7 & S / S; D e No & Sim & Deambulador & 71,79 & 100 & 90,47 & 86,45 \\
\hline 5 & 9 & 8 & S / S; D e No & Sim & Deambulador & 61,53 & 100 & 100 & 84,37 \\
\hline 6 & 10 & 9 & $\mathrm{~S} / \mathrm{N}$ & Sim & Deambulador & \multicolumn{4}{|c|}{ Não avaliado } \\
\hline 7 & 13 & 8 & $S / N$ & Não & Cadeirante & 2,56 & 58,33 & 66,66 & 37,50 \\
\hline 8 & 13 & 8 & $\mathrm{~S} / \mathrm{N}$ & Não & Cadeirante & 12,82 & 94,44 & 90,47 & 60,41 \\
\hline 9 & 13 & 8 & S / S; D e No & Sim & Deambulador & 92,3 & 100 & 100 & 96,87 \\
\hline 10 & 13 & 9 & $\mathrm{~S} / \mathrm{N}$ & Sim & Cadeirante & \multicolumn{4}{|c|}{ Não avaliado } \\
\hline $\begin{array}{l}\text { Média } \\
\text { (DP) }\end{array}$ & $\begin{array}{c}10,5 \\
(2,40)\end{array}$ & $\begin{array}{c}7,50 \\
(1,17)\end{array}$ & --- & --- & --- & $\begin{array}{c}48,71 \\
(33,00)\end{array}$ & $\begin{array}{c}92,35 \\
(14,14)\end{array}$ & $\begin{array}{c}89,28 \\
(11,59)\end{array}$ & $\begin{array}{c}73,95 \\
(19,82)\end{array}$ \\
\hline
\end{tabular}

DP: desvio-padrão; S: sim; N: não; D: diurna; No: noturna; MFM: Escala de Função Motora; D1, D2, D3: dimensões 1, 2 e 3 respectivamente

Tabela 2. Termos não compreendidos pelos pacientes

\begin{tabular}{|c|c|c|c|}
\hline $\begin{array}{l}\text { Termos não } \\
\text { compreendidos }\end{array}$ & $\begin{array}{c}\text { Pacientes }(P) \text { que não } \\
\text { compreenderam o termo }\end{array}$ & $\begin{array}{l}\text { Explicação adicional } \\
\text { ao termo* }\end{array}$ & $\begin{array}{l}\text { Pacientes (P) que não compreenderam o } \\
\text { termo mesmo após explicação adicional }\end{array}$ \\
\hline Náusea & P2, P3, P5, P7 e P9 & "tontura e enjoo" & P3, P5 e P7 \\
\hline Apetite & P3 & "apetite é a vontade de comer" & - \\
\hline Falta de ar & P4 & "dificuldade de respirar" & P4 \\
\hline Ansiedade & P5 & "sentir-se nervoso" & P5 \\
\hline
\end{tabular}

* explicação realizada apenas para os pacientes que não compreenderam inicialmente o termo

Tabela 3. Pontuação (média e desvio padrão) dos sintomas no Edmonton Symptom Assessment System (ESAS) e na Escala de Faces e análise de correlação

\begin{tabular}{|c|c|c|c|c|c|c|c|c|c|}
\hline & Dor & Cansaço & Sonolência & Náusea & Apetite & Falta de ar & Depressão & Ansiedade & Bem estar geral \\
\hline$E S A S-A^{a}$ & $\begin{array}{c}0,00 \\
(0,00)\end{array}$ & $\begin{array}{c}0,00 \\
(0,00)\end{array}$ & $\begin{array}{c}0,70 \\
(1,64)\end{array}$ & $\begin{array}{c}0,00 \\
(0,00)\end{array}$ & $\begin{array}{c}0,00 \\
(0,00)\end{array}$ & $\begin{array}{c}0,00 \\
(0,00)\end{array}$ & $\begin{array}{c}0,70 \\
(2,21)\end{array}$ & $\begin{array}{c}0,50 \\
(1,58)\end{array}$ & $\begin{array}{c}0,00 \\
(0,00)\end{array}$ \\
\hline ESAS - $\mathbf{P}^{a}$ & $\begin{array}{c}1,10 \\
(2,85)\end{array}$ & $\begin{array}{c}0,90 \\
(1,73)\end{array}$ & $\begin{array}{c}1,40 \\
(3,13)\end{array}$ & $\begin{array}{c}0,00 \\
(0,00)\end{array}$ & $\begin{array}{c}4,78 \\
(4,09)\end{array}$ & $\begin{array}{c}0,11 \\
(0,33)\end{array}$ & $\begin{array}{c}1,00 \\
(3,16)\end{array}$ & $\begin{array}{c}0,56 \\
(1,67)\end{array}$ & $\begin{array}{c}0,00 \\
(0,00)\end{array}$ \\
\hline Escala de Faces - $\mathrm{P}^{a}$ & $\begin{array}{c}0,60 \\
(1,35)\end{array}$ & $\begin{array}{c}1,20 \\
(2,15)\end{array}$ & $\begin{array}{c}2,80 \\
(3,43)\end{array}$ & $\begin{array}{c}0,29 \\
(0,76)\end{array}$ & $\begin{array}{c}4,00 \\
(4,24)\end{array}$ & $\begin{array}{c}1,56 \\
(3,13)\end{array}$ & $\begin{array}{c}2,00 \\
(3,53)\end{array}$ & $\begin{array}{c}1,56 \\
(3,28)\end{array}$ & $\begin{array}{c}1,80 \\
(3,82)\end{array}$ \\
\hline rho (1) & $*$ & 0,25 & 0,50 & $*$ & 0,57 & $-0,18$ & 0,64 & 0,65 & $*$ \\
\hline rho (2) & $*$ & $*$ & 0,82 & * & $*$ & * & 1.00 & 1.00 & $*$ \\
\hline
\end{tabular}

$\bar{a}$ valores apresentados em média e desvio padrão. P: paciente. A: avaliador. (rho) valor do coeficiente de correlação de Spearman obtido entre o resultado obtido no ESAS e o resultado da Escala de Faces Revisada (1) e resultado obtido entre ESAS respondido pelo paciente e avaliador (2); * não foi possível avaliar essa correlação devido ausência de variabilidade nas respostas

\section{DISCUSSÃO}

É de suma importância que os pacientes com DMD possam se beneficiar da abordagem dos Cuidados Paliativos, principalmente no que diz respeito à adequada avaliação e manejo dos sintomas. ${ }^{4}$ Nesse sentido, fazia-se necessário verificar se crianças e adolescentes com DMD entendiam os termos do ESAS e se eram capazes de graduar seus sintomas por meio deste instrumento, tão amplamente utilizado na prática do cuidado paliativo.

Os resultados aqui apresentados serão subsídios para a continuidade de outras pesquisas do nosso grupo de pesquisa envolvendo o uso do ESAS nesse perfil de paciente. O perfil funcional de pacientes com DMD, apesar de bem delineado, é considerado heterogêneo. ${ }^{1}$ Esta heterogeneidade é observada na amostra deste estudo, pois o escore total da MFM dos 8 pacientes que tinham essa avaliação variou entre $37,5 \%$ (P7) a $96,87 \%$ (P9). Diante dessa variabilidade, questionamos se existe relação entre a involução das habilidades motoras (avaliadas pela MFM) e o nível cognitivo/capacidade de compreensão dos pacientes.

A literatura não traz informações sobre essa questão e os resultados indicaram que os sintomas dor, cansaço, sonolência, depressão (tristeza) e bem-estar foram compreendidos por todos os pacientes. Houve dificuldade de compreensão de alguns pacientes para os sintomas falta de ar (P4), náusea (P2, P3, P5, P7 e P9), apetite (P3) e ansiedade (P5).

O termo "náusea" foi o que mais gerou dúvida entre os pacientes, visto que foi necessária a explicação para cinco deles (P2, P3, P5, P7 E P9). Não existem dados na literatura que 
possibilitem comparar esses achados com outros pacientes pediátricos, entretanto, dois estudos brasileiros em pacientes adultos em cuidados paliativos também relataram dificuldade quanto à compreensão deste termo, sendo necessária a inclusão de termos explicativos entre parênteses. ${ }^{9,21}$ Os resultados do presente estudo parecem indicar que, assim como na aplicação da escala em adultos, é necessário realizar uma explicação adicional do item para os pacientes com DMD.

Em relação aos termos "apetite" e "ansiedade" não foi possível realizar comparações devido ausência de estudos. O termo "falta de ar" não foi compreendido apenas por um paciente ( $\mathrm{P} 4)$ sendo que seu responsável afirmou que o mesmo nunca apresentou tal queixa.

A dispneia é uma experiência subjetiva e sua avaliação precisa depende do autorrelato do paciente. Dessa forma, a partir do relato do cuidador, infere-se que o desconhecimento do termo tenha relação com a falta de experiência da situação, uma vez que, a dispneia é um dos sintomas considerados mais assustadores pelas crianças e por seus responsáveis. ${ }^{25}$

Apesar dessas dificuldades na compreensão de alguns termos, todos os pacientes conseguiram graduar seus sintomas pelo ESAS e pela Escala de Faces, sendo que em ambos os instrumentos houve uma pontuação média baixa dos sintomas. Com exceção do apetite, a média de todos os sintomas foram abaixo de 2 no ESAS e abaixo de 3 na Escala de Faces.

É sabido que qualidade de vida na DMD está diretamente relacionada à função motora e que, as queixas físicas/emocionais podem tornar-se mais perceptíveis quando o paciente perde a deambulação e adota a cadeira de rodas continuamente. ${ }^{26}$ Assim, acredita-se que a baixa pontuação obtida pelos pacientes seja devido ao perfil da amostra que é jovem (média de 10,5 anos) e com alto escore funcional (média de $72,4 \%$ escore total da MFM).

Sob a ótica do avaliador, a maior parte dos sintomas foi graduado como intensidade "zero". De modo geral, os pacientes tenderam a avaliar os seus sintomas como mais intensos que $o$ avaliador.

Embora o padrão ouro seja o preenchimento do ESAS pelo autorrelato do paciente, também pode ser preenchido pelo profissional de saúde de acordo com a sua percepção dos sintomas apresentados pelo paciente. ${ }^{8}$

Existe um alto nível de concordância e pontuação entre os sintomas relados pelo paciente e os sintomas observados por médicos. ${ }^{27} \mathrm{E}$, portanto, essa questão ainda precisa ser melhor explorada em pacientes com DMD, colhendo dados do ESAS tanto em pacientes quanto em avaliadores e cuidadores.

Apesar deste estudo ter algumas limitações como pequeno tamanho amostral e ausência de dados sobre o nível cognitivo dos pacientes, os dados podem ser considerados inovadores por ser a primeira vez que o ESAS é aplicado em pacientes com DMD. Ainda que de maneira incipiente, esses achados podem contribuir na inserção da abordagem paliativa para esse perfil de paciente.

\section{CONCLUSÃO}

Não há necessidade de alteração dos termos do ESAS-r para avaliação de sintomas de pacientes com DMD, visto que esses pacientes entenderam os termos e foram capazes de graduar seus sintomas por meio deste instrumento. Para tal e adequado uso desse instrumento na prática clínica, sugere-se que os dados desse estudo sejam utilizados para direcionar um treinamento de profissionais da saúde.

\section{Agradecimentos}

Agradecimentos a toda a equipe do Laboratório de Estrutura e Função do Músculo Esquelético no qual esse estudo é desenvolvido; aos pacientes e seus responsáveis pelo consentimento na participação da pesquisa, à FAPESP (Fundação de Amparo à Pesquisa do Estado de São Paulo) pelo financiamento desse projeto (Processo n. 2019/15408-1), ao CNPq (Conselho Nacional de Desenvolvimento Científico e Tecnológico) pela bolsa de Produtividade em Pesquisa e à Capes pelo fomento junto ao Programa de Pós-Graduação em Reabilitação e Desempenho Funcional.

\section{REFERÊNCIAS}

1. Humbertclaude V, Hamroun D, Bezzou K, Bérard C, Boespflug-Tanguy $\mathrm{O}$, Bommelaer $\mathrm{C}$, et al. Motor and respiratory heterogeneity in Duchenne patients: Implication for clinical trials. Eur J Paediatr Neurol. 2012;16(2):149-60.

Doi: https://doi.org/10.1016/i.ejpn.2011.07.001

2. Silva TDD, Massetti T, Monteiro CBDM, Trevizan IL, Arab $C$, Caromano FA, et al. Pain characterization in Duchenne muscular dystrophy. Arquivos de neuropsiquiatria. 2016;74(9),767-74. Doi: http://dx.doi.org/10.1590/0004-282X20160107

3. Pangalila RF, van den Bos GA, Bartels B, Bergen $M$, Stam HJ, Roebroeck ME. Prevalence of fatigue, pain, and affective disorders in adults with duchenne muscular dystrophy and their associations with quality of life. Arch Phys Med Rehabil. 2015;96(7):1242-7. Doi: https://doi.org/10.1016/j.apmr.2015.02.012

4. Carter GT, Joyce NC, Abresch AL, Smith AE, VandeKeift GK. Using palliative care in progressive neuromuscular disease to maximize quality of Life. Phys Med Rehabil Clin N Am. 2012;23(4):903-9.

Doi: http://dx.doi.org/10.1016/j.pmr.2012.08.002

5. Carvalho RT, Parsons, HA. Manual de cuidados paliativos ANCP: ampliado e atualizado. São Paulo: Academia Nacional de Cuidados Paliativos (ANCP); 2012.

6. Organización Mundial de la Salud. Cuidados paliativos [texto na Internet]. Geneva: WHO; c2020 [citado 2020 Jun 21]. Disponível em: https://www.who.int/es/newsroom/fact-sheets/detail/palliative-care

7. Rushton $\mathrm{CH}$, Erby $\mathrm{LH}$, Cohn RD, Geller G. Integrating palliative care in life-limiting pediatric neuromuscular conditions: the case of SMA-type 1 and Duchene muscular dystrophy. J Palliat Care Med. 2012; 2(1), 103. Doi: http://dx.doi.org/10.4172/2165-7386.1000103

8. Bruera E, Kuehn N, Miller MJ, Selmser P, Macmillan K. The Edmonton Symptom Assessment System (ESAS): a simple method for the assessment of palliative care patients. J Palliat Care. 1991;7(2):6-9. Doi: https://doi.org/10.1177/082585979100700202 
9. Paiva CE, Manfredini LL, Paiva BS, Hui D, Bruera E. The Brazilian Version of the Edmonton Symptom Assessment System (ESAS) Is a Feasible, Valid and Reliable Instrument for the Measurement of Symptoms in Advanced Cancer Patients. PLoS One. 2015 8;10(7):e0132073. Doi: https://doi.org/10.1371/journal.pone.0132073

10. Davison SN, Jhangri GS, Johnson JA. Longitudinal validation of a modified Edmonton symptom assessment system (ESAS) in haemodialysis patients. Nephrol Dial Transplant. 2006;21(11):3189-95.

Doi: https://doi.org/10.1093/ndt/gfl380

11. Shah AB, Udeoji DU, Baraghoush $A$, Bharadwaj $P$, Yennurajalingam $S$, Schwarz ER. An evaluation of the prevalence and severity of pain and other symptoms in acute decompensated heart failure. J Palliat Med. 2013;16(1):87-90.

Doi: https://doi.org/10.1089/jpm.2012.0248

12. Walke LM, Gallo WT, Tinetti ME, Fried TR. The burden of symptoms among community-dwelling older persons with advanced chronic disease. Arch Intern Med. 2004; 164 (21): 2321-24. Doi: https://doi.org/10.1001/archinte.164.21.2321

13. Poonja Z, Brisebois A, van Zanten SV, Tandon P, Meeberg G, Karvellas CJ. Patients with cirrhosis and denied liver transplants rarely receive adequate palliative care or appropriate management. Clin Gastroenterol Hepatol. 2014;12(4):692-8.

Doi:

https://doi.org/10.1016/j.cgh.2013.08.027

14. Lopez G, Liles DK, Knupp CL. Edmonton Symptom Assessment System for outpatient symptom monitoring of sickle cell disease. South Med J. 2014;107(12):768-72. Doi: https://doi.org/10.14423/SMJ.0000000000000209

15. Hunt A, Carter B, Abbott J, Parker A, Spinty S, deGoede C. Pain experience, expression and coping in boys and young men with Duchenne muscular dystrophy - a pilot study using mixed methods. Eur J Paediatr Neurol. 2016;20(4):630-8.

Doi: https://doi.org/10.1016/j.ejpn.2016.03.002

16. El-Aloul B, Speechley KN, Wei Y, Wilk P, Campbell C. Fatigue in young people with Duchenne muscular dystrophy. Dev Med Child Neurol. 2020;62(2):245-51. Doi: https://doi.org/10.1111/dmcn.14248

17. Toussaint $M$, Soudon $P$, Kinnear W. Effect of non-invasive ventilation on respiratory muscle loading and endurance in patients with Duchenne muscular dystrophy. Thorax. 2008;63(5):430-4.

Doi:
18. Ferreira, VR. Escala de distúrbios do sono em crianças: tradução, adaptação cultural e validação [Dissertação]. São Paulo: Universidade Federal de São Paulo; 2009.

19. Evans A, Vingelen MB, Yu C, Baird J, Murray P, Bryant P. Nausea in numbers: electronic medical record nausea and vomiting assessment for children with cancer. J Pediatr Oncol Nurs. 2020;37(3):195-203. Doi: https://doi.org/10.1177/1043454219900467

20. Damani A, Salins N, Ghoshal A, Muckaden M. Specialist Pediatric Palliative Care Prescribing Practices: A Large 5year Retrospective Audit. Indian J Palliat Care. 2016;22(3):274-281. Doi: https://doi.org/10.4103/09731075.185033

21. Monteiro DR, Almeida MA, Kruse MHL. Tradução e adaptação transcultural do instrumento Edmonton Symptom Assessment System para uso em Cuidados Paliativos. Rev Gaúcha Enferm. 2013;34(2):163-71. Doi: https://doi.org/10.1590/S1983-14472013000200021

22. Silva FC, Thuler LC. Cross-cultural adaptation and translation of two pain assessment tools in children and adolescents. J Pediatr (Rio J). 2008;84(4):344-9. Doi: https://doi.org/10.2223/JPED.1809

23. Iwabe C, Miranda-Pfeilsticker BH, Nucci A. Medida da função motora: versão da escala para o português e estudo de confiabilidade. Rev Bras Fisioter. 2008;12(5):417-24. Doi: https://doi.org/10.1590/S141335552008000500012

24. Pagano M, Gauvreau K. Principles of biostatistics. New York: CRC; 2018.2 Doi: https://doi.org/10.1201/9780429489624

25. Craig F, Henderson EM, Bluebond-Langner M. Management of respiratory symptoms in paediatric palliative care. Curr Opin Support Palliat Care.2015;9(3):217-26. Doi: https://doi.org/10.1097/SPC.0000000000000154

26. Gocheva V, Schmidt S, Orsini AL, Hafner P, Schaedelin S, Rueedi $\mathrm{N}$, et al. Association between health-related quality of life and motor function in ambulant and nonambulant Duchenne muscular dystrophy patients. J Child Neurol. 2019;34(14):873-85. Doi: https://doi.org/10.1177/0883073819865681

27. Brooks JV, Poague C, Formagini T, Sinclair CT, NelsonBrantley HV. The Role of a Symptom Assessment Tool in Shaping Patient-Physician Communication in Palliative Care. J Pain Symptom Manage. 2020;59(1):30-8. Doi: https://doi.org/10.1016/j.jpainsymman.2019.08.024 